Article

\title{
Di-Chromatic InGaN Based Color Tuneable Monolithic LED with High Color Rendering Index
}

\author{
Amit Yadav ${ }^{1, *(1)}$, Ilya E. Titkov ${ }^{2}$, Alexei V. Sakharov ${ }^{3}$, Wsevolod V. Lundin ${ }^{3}$, \\ Andrey E. Nikolaev ${ }^{3}$, Grigorii S. Sokolovskii ${ }^{3,4}$, Andrey F. Tsatsulnikov ${ }^{3}$ and \\ Edik U. Rafailov ${ }^{1}$ (D) \\ 1 Optoelectronics and Biomedical Photonics Group, AIPT, Aston University, Birmingham B4 7ET, UK; \\ e.rafailov@aston.ac.uk \\ 2 Nano-Technology Lab., Ostendo Technologies Inc., 6185 Paseo del Norte, Carlsbad, CA 92011, USA; \\ ilya.titkov@ostendo.com \\ 3 Physics of Semiconductor Heterostructure Lab., Ioffe Institute, 26 Politekhnicheskaya Str., \\ 194021 St. Petersburg, Russia; val@beam.ioffe.ru (A.V.S.); Lundin@vpegroup.ioffe.ru (W.V.L.); \\ Aen@mail.ioffe.ru (A.E.N.); gs@mail.ioffe.ru (G.S.S.); Andrew@beam.ioffe.ru (A.F.T.) \\ 4 Optoelectronics Department, St. Petersburg State Electrotechnical University (LETI), 5 Prof. Popova Str., \\ 197022 St. Petersburg, Russia \\ * Correspondence: a.yadav1@aston.ac.uk; Tel.:+44-121-204-3703
}

Received: 23 May 2018; Accepted: 13 July 2018; Published: 17 July 2018

\begin{abstract}
We demonstrate a phosphor free, dichromatic GaN-based monolithic white LED with vertically stacked green and blue emitting multiple quantum wells. The optimal thickness of GaN barrier layer between green and blue quantum wells used is $8 \mathrm{~nm}$. This device can be tuned over a wide range of correlated color temperature (CCT) to achieve warm white (CCT $=3600 \mathrm{~K})$ to cool white $(\mathrm{CCT}=13,000 \mathrm{~K})$ emission by current modulation from $2.3 \mathrm{~A} / \mathrm{cm}^{2}$ to $12.9 \mathrm{~A} / \mathrm{cm}^{2}$. It is also demonstrated for the first time that a color rendering index (CRI) as high as 67 can be achieved with such a dichromatic source. The observed CCT and CRI tunability is associated with the spectral power evolution due to the pumping-induced carrier redistribution.
\end{abstract}

Keywords: monolithic LED; high CRI; light-emitting diodes; color rendering; colorimetry

\section{Introduction}

White LEDs based on InGaN are used in many applications from backlighting to mobile displays and general illumination [1-3]. The requirements on characteristic and quality of white light emission differ with application. While phosphor covered blue LEDs with improved color rendering index (CRI) and luminous efficacy are replacing traditional light sources [3] at offices, museum and similar application areas, high CRI is irrelevant for indicator and signage. For outdoor street lighting, industrial use and parking spaces sources with CRI $\geq 60$ and $C T \leq 8000 \mathrm{~K}$ are considered adequate [3-5]. Hence, white light source with tunable CRI and CCT are desirable. Towards this, a multichip approach with vertical or lateral combination of blue, green and red LEDs can be used for white light generation. However, this approach requires complex fabrication procedures, driving circuits, and device design, thereby affecting reliability and increasing production cost [2]. White light emission has also been achieved with CdSe/ZnS nanocrystals and by doping of InGaN quantum wells (QWs) with Si and $\mathrm{Zn}[6,7]$. These approaches along with the shortcomings of the multichip approach have an additional disadvantage of non-tunability. Meanwhile, the theoretical possibility of emission from $0.7 \mathrm{eV}$ to $3.5 \mathrm{eV}$ by indium (In) variation in InGaN QWs, is also being explored [8-11]. This all semiconductor monolithic approach holds potential of efficient color tunable sources with high CRI. 
Monolithic approaches involving nanostructure engineering has been explored previously for white light emission. A dual wavelength, $5000 \mathrm{~K}$ to 20,000 K color tunable, multifacet QW LED was demonstrated by Funato et al. in 2008 [12]. Nguyen et al. have reported color tunability and CRI values more than 90 for "dot in a wire" core shell LEDs on silicon [13]. Nevertheless, this approach requires a complex growth and fabrication process with precise control over wire diameter and dot size, which is not ideal for mass production. In addition, luminous efficacy of these devices is still far from phosphor covered LEDs [13]. Li et al. demonstrated dual wavelength MQW LED with $46 \%$ indium (In) content for red emission peak at $2.12 \mathrm{eV}$ thus achieving emission color from red to yellow to white with CRI of 85.6 [14]. However, large lattice mismatch between higher In incorporated InGaN red QW on GaN substrate results in issues related to charge separation and increased defect density [15]. A simpler fabrication approach of vertically stacked QWs emitting at two distinct wavelengths has been reported. Active region of such devices can consist of either: (i) longer wavelength emitting passive QWs pumped by active blue QW [16]; or (ii) all electrically pumped active QWs $[8,11,17]$. The first approach is similar to phosphor covered LEDs and the spectrum is controlled by number of passive QWs in active region. In addition, since the passive QWs are designed to operate on the green gap spectral range they being less efficient than phosphor for that range and their sensitivity to active QW emission wavelength is a disadvantage [18]. The best CRI for such devices reported is 41 [16]. White light emission from all electrically pumped QWs with CCT $6000 \mathrm{~K}$ has been reported previously [19].

In this paper, we demonstrate phosphor free, color tunable monolithic white LED. The device is designed for dichromatic emission at $450 \mathrm{~nm}$ and $550 \mathrm{~nm}$. The emission wavelengths are such chosen so that the line joining the corresponding $x-y$ coordinates on CIE 1931 chromaticity diagram passes through the white region. The $x-y$ tristimulus coordinates of standard D65 illuminant is demonstrated with these devices at current densities of $6.21 \mathrm{~A} / \mathrm{cm}^{2}$. It is also shown that CRI of over 60 can be demonstrated with these devices and CCT of emission in such LEDs can be easily tuned by modulation of pumping current.

\section{Device Structure and Experiment}

The InGaN-based LED samples investigated in this study were grown using MOCVD on c-plane sapphire. A Si-doped n-GaN layer is grown on buffer GaN layer. Short period superlattice (SPSL) with following low-temperature grown i-GaN barrier layer is grown over the $\mathrm{n}-\mathrm{GaN}$ to improve emission efficiency as was shown both for blue and green LEDs [20,21]. The SPSL are grown, as described by Lundin et al., by conversion method [21]. The SPSL has 12 alternate iterations of $1 \mathrm{~nm}$ thick InGaN and $\mathrm{GaN}$. This is followed with a $12 \mathrm{~nm}$ intrinsic GaN barrier layer.

Two blue QWs emitting at 440-450 nm were grown on top of i-GaN barrier layer followed by single green QW (540-570 nm) with spatial separation of $8 \mathrm{~nm}$ among the wells. An $18 \mathrm{~nm}$ AlGaN electron blocking layer (EBL) and $180 \mathrm{~nm} \mathrm{p-GaN} \mathrm{layer} \mathrm{is} \mathrm{deposited} \mathrm{on} \mathrm{top} \mathrm{of} 8 \mathrm{~nm}$ i-GaN barrier after the green QW. Figure 1 depicts the schematic of the conduction band diagram for the as grown devices. The structure was processed as $1310 \times 1310 \mu \mathrm{m}^{2}$ flip-chips, without epoxy, with design and optical properties described elsewhere [22].

The absolute values of the radiometric parameters, namely EQE, CCT and CRI, at room temperature were measured using an integration sphere and Labsphere CDS-600 spectrometer (Labsphere, Inc., Sutton, NH, USA) with "LightMtrx" software. As we were interested in studying evolution of optical parameters over a wide range of pumping, a Keithley2400 source (Tektronix, Inc., Beaverton, OR, USA) was used to supply current from as low as $20 \mathrm{~mA}$ up to $1 \mathrm{~A}$. Exposure time was varied from $1 \mathrm{~ms}$ to $5000 \mathrm{~ms}$ to record the absolute values of radiant flux from $1 \mathrm{nW}$ to $100 \mathrm{~mW}$. To obtain green/blue integrated intensity ratio, we measured blue and green part of the emission spectra separately by adjusting the digital apertures. 


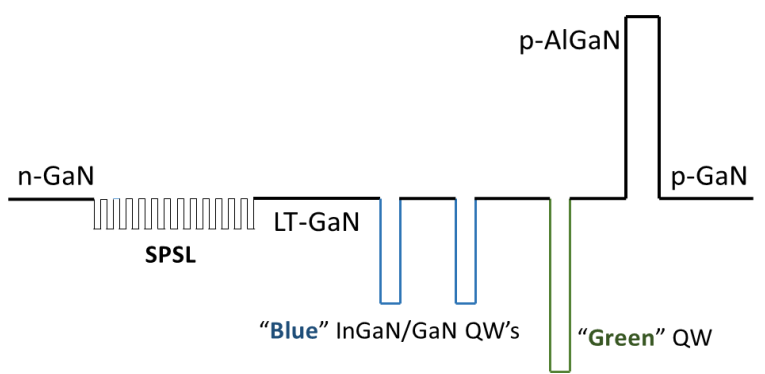

Figure 1. Conduction band schematic for the monolithic white LEDs depicting blue and green quantum wells (QWs) with bottom SPSL grown on top of $\mathrm{n}-\mathrm{GaN}$ in order to facilitate carrier injection and improve growth.

\section{Results}

The current-voltage (I-V) characteristic under CW regime at $300 \mathrm{~K}$ was studied for the LEDs from the same wafer. The I-V curve was fitted with a parallel and series resistance for leakage analysis. The obtained parameters are: $170 \mathrm{MOhm}$ parallel resistance, ideality factor of 4.3 , series resistance of $25 \mathrm{Ohm}$, and saturation current of $1.5 \times 10^{-14}$ A. The electroluminescence (EL) performance of monolithic white LEDs was studied at room temperature in continuous wave (CW) regime. Figure 2 shows EL spectra for these devices with increasing currents up to $500 \mathrm{~mA}$. The further increase of current was limited by the damage of p-n junction due to due overheating under CW regime, especially for longer durations. Two distinct peaks in Figure 2 indicate that both shallow and deep QWs are operating in their respective blue and green spectral regions. Figure 2a shows that at lower currents less than $80 \mathrm{~mA}$ the green peak dominates the emission spectrum with blue emission getting stronger with increasing carrier concentration. We attribute this to the non-uniform distribution of injected holes. Primarily, due to their lower mobility, higher effective mass, and poor transport among the MQW structure [23] holes will radiatively recombine in the QW closer to the p-side. However, with further increase in current, more holes travel through the barrier layer and are available for recombination in blue QWs. In MQW structures, non-uniform hole redistribution leading to dominant EL intensity from the QW at lower currents has been observed. Methods such as quaternary electron blocking layer [23], InGaN barrier layers [24] and varying thickness of the barrier layers [25] have been proposed to achieve better hole injection and transport among the QWs.
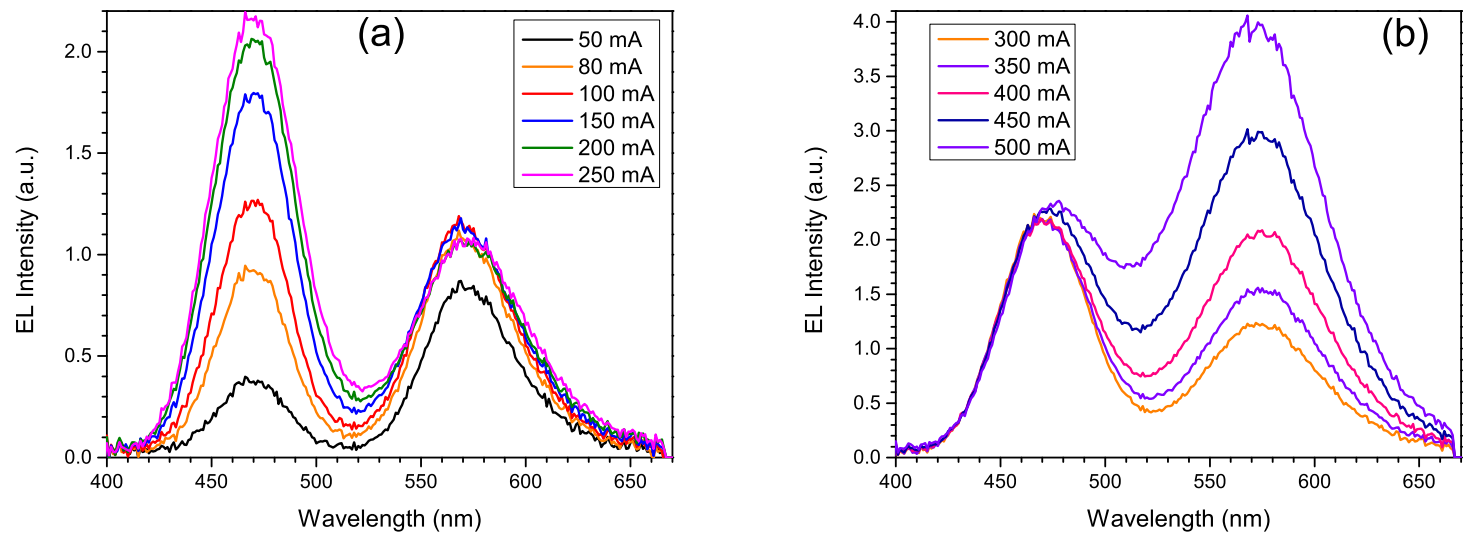

Figure 2. Electroluminescence (EL) spectra of monolithic white LED pumped with the continuous wave (CW) current of: (a) 50-250 mA; and (b) 300-500 mA. 
On further increasing the current up to $100 \mathrm{~mA}$, the emission from green $\mathrm{QW}$ is clamped and the radiative recombination in the blue $\mathrm{QW}$ is enhanced. For very high $\mathrm{CW}$ current injection, i.e., $>300 \mathrm{~mA}$, increased radiative recombination in green QW and saturated blue emission peak is observed (Figure 2b). This is believed to be caused by the carrier redistributing due to band-filling of blue QW leading to electron overflow thus making more carriers, i.e., electron available for recombination in green $\mathrm{QW}$. The EQE for these devices exhibit a dome-like dependence on current characteristic similar to mono-color InGaN LEDs. The EQE for devices tested from different parts of the wafer varied from below $1 \%$ to $4 \%$. This can be further improved by catering for procedures, during growth and fabrication, to improve light extraction efficiency. To correctly estimate and improve the internal quantum efficiency more studies concerning the crystalline structure, Indium composition, distance between QWs and p-doped region are required.

The tuning of CCT with varying current is shown in Figure 3. The dashed red line associates the highest CCT obtained from this device to the minima of green/blue $(\mathrm{G} / \mathrm{B})$ integrated intensity ratio. This indicates the dominance of blue peak in this region of operation. For injection current between $100 \mathrm{~mA}$ and $350 \mathrm{~mA}, \mathrm{G} / \mathrm{B}$ ratio is $<1$ and blue peak dominates resulting in cool white emission. For other regions, increase in green intensity results in tuning of emission towards warmer color temperature.

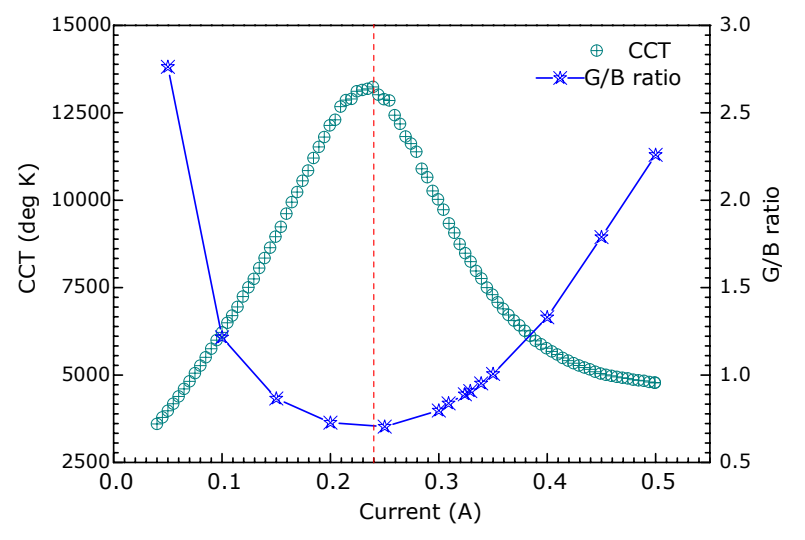

Figure 3. Green/Blue (G/B) ratio and corresponding correlated color temperature (CCT) tuning with variation of direct current.

Commission International de I'Elairage (CIE 1931) chromaticity coordinates with associated CCT at different currents is shown in Figure 4. The coordinates $(0.4172,0.4375)$ at $40 \mathrm{~mA}$ moves to $(0.2686,0.2716)$ at $240 \mathrm{~mA}$ and CCT increase from $3600 \mathrm{~K}$ to $13,240 \mathrm{~K}$ (Figure 4a). With further increase in current, a movement towards warmer CCT values can be seen with $4775 \mathrm{~K}$ at $(0.3607,0.4278)$ for $500 \mathrm{~mA}$. This distinctly depicts an excellent warm to cool white CCT tunable, simple monolithic LED. The CRI for device under test is under 40 for less than $70 \mathrm{~mA}$; however, with increasing carrier density, the spectral broadening of the green peak is asymmetric and spectral emission contribution at longer wavelengths (Figure 2b) greater than $600 \mathrm{~nm}$ improves the visible spectral region coverage thus improving the CRI values to greater than 60 with a maximum of 67.3 at $335 \mathrm{~mA}$ ever reported for such devices (Figure 5a). 

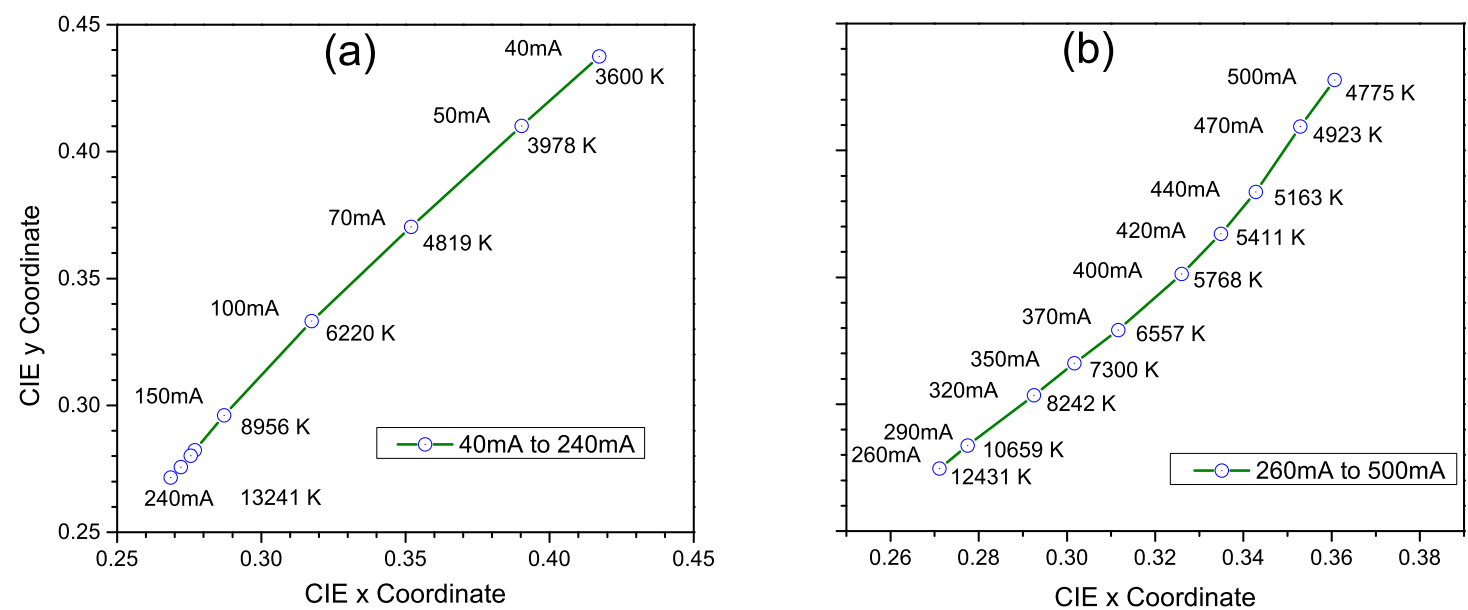

Figure 4. Commission International de I'Elairage (CIE) Chromaticity coordinates and corresponding CCT at various injection currents: (a) current from $40 \mathrm{~mA}$ to $240 \mathrm{~mA}$; and (b) current from $260 \mathrm{~mA}$ to $500 \mathrm{~mA}$.
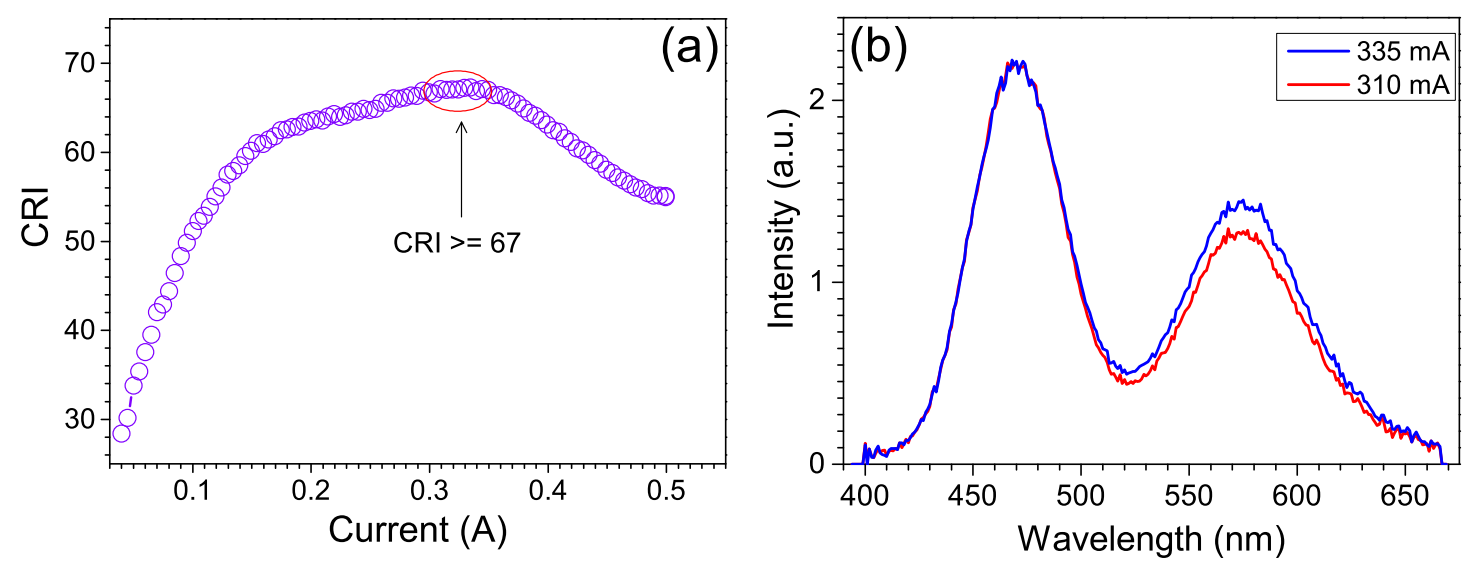

Figure 5. (a) Evolution of color rendering index (CRI) with increasing current; and (b) EL Spectrum at current values with CRI $\geq 67$ with a maximum of 67.3 at $335 \mathrm{~mA}$.

A further increase in current broadens the green peak. This broadening is asymmetric with increased spectral emission at shorter wavelengths. This overall broadening of green peak can be attributed to band-filling and screening of quantum confined stark effect at higher currents. A red shift of $7 \mathrm{~nm}$ in peak wavelength for blue emission is observed for current $>300 \mathrm{~mA}$ up to $500 \mathrm{~mA}$ which otherwise remains at $\sim 469 \mathrm{~nm}$. This is generally indicative of increase in junction temperature (see Figure 2b). On the other hand, a decrease in CRI for current $>350 \mathrm{~mA}$ (Figure 5a) can be explained by the change in the green/blue $(\mathrm{G} / \mathrm{B})$ spectral power density ratio and shift in the peak emission wavelength for both blue and green QW's. This indicates that, apart from broadened spectra, CRI in such devices can be sensitive to G/B ratio, since maximum CRI is obtained for G/B ratio between 0.84 and 1.5 (six closely spaced green stars in Figure 3). Spectrum of this region is shown in Figure $5 \mathrm{~b}$.

From the point of view of practical applications, the current dependence of the LED color characteristics is an undesirable factor, as additional current control is required for their stabilization. On the other hand, this effect opens additional opportunity for controlling the color parameters of monolithic LEDs by the current variation. The way to control this is pulsed operation with different current amplitude and durations. With another LED chip taken from the same wafer, we demonstrate that green/blue ratio strongly depends on the characteristics of pulses (Figure 6a) for the blue-green LED structures. Adjusting of the pulse regime allows changing the green/blue ratio keeping luminous 
flux constant (Figure 6b). This effect leads to change of color coordinates of the emission in a wide enough range. Different spectral behavior from the first chip can be explained by its high sensitivity to the GaN barrier thickness [18].
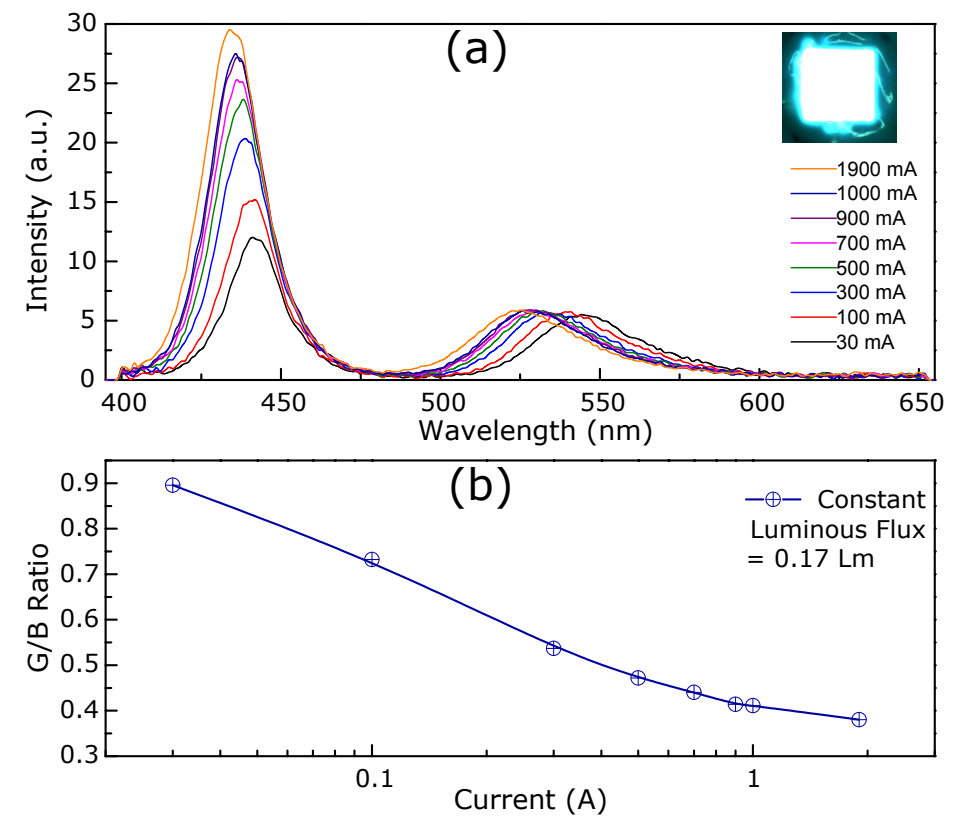

Figure 6. (a) Electroluminescence (EL) spectra of the MBG LEDs under pulsed excitation, pulse width changed from $17 \mu$ s at low current amplitude ( $30 \mathrm{~mA}$ ) to $0.58 \mu$ s at high current amplitude (1.9 A), fixed period $=100 \mu \mathrm{s}$ (Inset: MBG LED chip. White light emission under pulsed current operation with 1.9 A peak current; the EL emission is collected from the back surface of the chip through sapphire substrate); (b) The dependence of integrated intensities of green and blue peaks on current at pulsed excitation and constant luminous flux.

\section{Conclusions}

In summary, we have demonstrated, for the first time to the best of our knowledge, phosphor free white light emission with CRI values as high as 67 from dichromatic QW LED structure emitting at blue and green wavelengths. We have also shown that it is possible to tune these devices from warm to cool white emission by variation of pumping current. It is observed that the CRI is sensitive to spectral broadening and the ratio of intensities of the green and blue peaks (G/B ratio). Further, it is demonstrated that the duty cycle variation in the pulse regime operation can be used to tailor $\mathrm{G} / \mathrm{B}$ ratio maintaining a constant luminous flux while simultaneously tuning the color coordinates of emission in wide range.

Author Contributions: All authors have contributed equally towards this work and the final revision of the manuscript.

Funding: This research was funded by EU Seventh Framework Program (NEWLED project) with Grant No. 318388.

Conflicts of Interest: The authors declare no conflict of interest.

\section{References}

1. Schubert, E.F.; Kim, J.K. Solid-State Light Sources Getting Smart. Science 2005, 308, 1274-1278.

2. Crawford, M. LEDs for Solid-State Lighting: Performance Challenges and Recent Advances. IEEE J. Sel. Top. Quantum Electr. 2009, 15, 1028-1040. [CrossRef] 
3. Cree Inc. Cree First to Break 300 Lumens-Per-Watt Barrier. 2014. Available online: http://www.cree. com/News-and-Events /Cree-News/Press-Releases/2014/March/300LPW-LED-barrier (accessed on 16 July 2018).

4. Schubert, E.F. Light-Emitting Diodes, 2nd ed.; Cambridge University Press: Cambridge, UK, 2006.

5. Tsao, J.Y.; Crawford, M.H.; Coltrin, M.E.; Fischer, A.J.; Koleske, D.D.; Subramania, G.S.; Wang, G.T.; Wierer, J.J.; Karlicek, R.F. Toward Smart and Ultra-Efficient Solid—State Lighting. Adv. Opt. Mater. 2014, 2, 809-836. [CrossRef]

6. Nizamoglu, S.; Ozel, T.; Sari, E.; Demir, H.V. White light generation using CdSe/ZnS core-Shell nanocrystals hybridized with InGaN/GaN light emitting diodes. Nanotechnology 2007, 18, 065709. [CrossRef]

7. Chang, S.; Wu, L.; Su, Y.; Kuo, C.; Lai, W.; Hsu, Y.; Sheu, J.; Chen, J.; Tsai, J. White light generation using CdSe/ZnS core-shell nanocrystals hybridized with InGaN/GaN light emitting diodes. IEEE Trans. Electr. Devices 2003, 50, 519-521. [CrossRef]

8. Damilano, B.; Grandjean, N.; Pernot, C.; Massies, J. Monolithic white light emitting diodes based on InGaN/GaN multiple-quantum wells. Jpn. J. Appl. Phys. 2001, 40, L918. [CrossRef]

9. Yamada, M.; Narukawa, Y.; Mukai, T. Phosphor free high-luminous-efficiency white light-emitting diodes composed of InGaN multi-quantum well. Jpn. J. Appl. Phys. 2002, 41, L246. [CrossRef]

10. Lu, C.F.; Huang, C.F.; Chen, Y.S.; Shiao, W.Y.; Chen, C.Y.; Lu, Y.C.; Yang, C.C. Phosphor-free monolithic white-light LED. IEEE J. Sel. Top. Quantum Electr. 2009, 15, 1210-1217.

11. Tsatsulnikov, A.F.; Lundin, W.V.; Sakharov, A.V.; Zavarin, E.E.; Usov, S.O.; Nikolaev, A.E.; Sizov, V.S.; Zakgeim, A.L.; Mizerov, M.N.; Cherkashin, N.A.; et al. Effect of stimulated phase separation on properties of blue, green and monolithic white LEDs. Phys. Status Solidi C 2012, 9, 774-777. [CrossRef]

12. Funato, M.; Hayashi, K.; Ueda, M.; Kawakami, Y.; Narukawa, Y.; Mukai, T. Emission color tunable light-emitting diodes composed of InGaN multifacet quantum wells. Appl. Phys. Lett. 2008, 93, 021126. [CrossRef]

13. Nguyen, H.P.T.; Zhang, S.; Cui, K.; Han, X.; Fathololoumi, S.; Couillard, M.; Botton, G.A.; Mi, Z. p-Type modulation doped InGaN/GaN dot-in-a-wire white-light-emitting diodes monolithically grown on Si (111). Nano Lett. 2011, 11, 1919-1924. [CrossRef] [PubMed]

14. Li, H.; Li, P.; Kang, J.; Li, Z.; Li, Z.; Li, J.; Yi, X.; Wang, G. Phosphor-free, color-tunable monolithic InGaN light-Emitting diodes. Appl. Phys. Express 2013, 6, 102103. [CrossRef]

15. Tan, C.-K.; Tansu, N. Nanostructured lasers: Electrons and holes get closer. Nat. Nanotechnol. 2016, 10, 107-109. [CrossRef] [PubMed]

16. Damilano, B.; Demolon, P.; Brault, J.; Huault, T.; Natali, F.; Massies, J. Blue-green and white color tuning of monolithic light emitting diodes. J. Appl. Phys. 2010, 108, 073115. [CrossRef]

17. Titkov, I.E.; Yadav, A.; Zerova, V.L.; Zulonas, M.; Tsatsulnikov, A.F.; Lundin, W.V.; Sakharov, A.V.; Rafailov, E.U. Internal Quantum Efficiency and Tunable Colour Temperature in Monolithic White InGaN/GaN LED; SPIE: San Francisco, CA, USA, 2014.

18. Karpov, S.Y.; Cherkashin, N.A.; Lundin, W.V.; Nikolaev, A.E.; Sakharov, A.V.; Sinitsin, M.A.; Usov, S.O.; Zavarin, E.E.; Tsatsulnikov, A.F. Multi-color monolithic III-nitride light-emitting diodes: Factors controlling emission spectra and efficiency. Phys. Status Solidi A 2016, 213, 19-29. [CrossRef]

19. Tsatsulnikov, A.F.; Lundin, W.V.; Sakharov, A.V.; Zavarin, E.E.; Usov, S.O.; Nikolaev, A.E.; Kryzhanovskaya, N.V.; Synitsin, M.A.; Sizov, V.S.; Zakgeim, A.L.; et al. A monolithic white LED with an active region based on InGaN QWs separated by short-period InGaN/GaN superlattices. Semiconductors 2010, 44, 808-811. [CrossRef]

20. Tsatsulnikov, A.F.; Lundin, W.V.; Sakharov, A.V.; Zavarin, E.E.; Usov, S.O.; Nikolaev, A.E.; Cherkashin, N.A.; Ber, B.Y.; Kazantsev, D.Y.; Mizerov, M.N.; et al. Active region based on graded-gap InGaN/GaN superlattices for high-power 440- to 470-nm light-emitting diodes. Semiconductors 2010, 44, 93-97. [CrossRef]

21. Lundin, W.V.; Nikolaev, A.E.; Sakharov, A.V.; Zavarin, E.E.; Valkovskiy, G.A.; Yagovkina, M.A.; Usov, S.O.; Kryzhanovskaya, N.V.; Sizov, V.S.; Brunkov, P.N.; et al. Single quantum well deep-green LEDs with buried InGaN/GaN short-period superlattice. J. Cryst. Growth 2011, 315, 267-271. [CrossRef]

22. Chernyakov, A.E.; Bulashevich, K.A.; Karpov, S.Y.; Zakgeim, A.L. Experimental and theoretical study of electrical, thermal, and optical characteristics of InGaN/GaN high-power flip-chip LEDs. Phys. Status Solidi A 2013, 210, 466-469. [CrossRef] 
23. Kim, J.; Ji, M.H.; Detchprohm, T.; Dupuis, R.D.; Shervin, S.; Ryou, J.H. Effect of lattice-matched InAlGaN electron-blocking layer on hole transport and distribution in InGaN/GaN multiple quantum wells of visible light-emitting diodes. Phys. Status Solidi A 2016, 213, 1296-1301. [CrossRef]

24. Kuo, Y.K.; Chang, J.Y.; Tsai, M.C.; Yen, S.H. Advantages of blue InGaN multiple-quantum well light-emitting diodes with InGaN barriers. Appl. Phys. Lett. 2009, 95, 011116. [CrossRef]

25. Liu, J.P.; Ryou, J.H.; Dupuis, R.D.; Han, J.; Shen, G.D.; Wang, H.B. Barrier effect on hole transport and carrier distribution in In Ga N/Ga N multiple quantum well visible light-emitting diodes. Appl. Phys. Lett. 2008, 93, 021102. [CrossRef]

(C) 2018 by the authors. Licensee MDPI, Basel, Switzerland. This article is an open access article distributed under the terms and conditions of the Creative Commons Attribution (CC BY) license (http://creativecommons.org/licenses/by/4.0/). 